\title{
Stark-Zeeman Line Shape Modeling for Magnetic White Dwarf and Tokamak Edge Plasmas: Common Challenges
}

\author{
Joël Rosato ${ }^{1, *}$, Ny Kieu ${ }^{1}$, Ibtissem Hannachi ${ }^{1}$, Mohammed Koubiti ${ }^{1}$, Yannick Marandet ${ }^{1}$, \\ Roland Stamm ${ }^{1}$, Milan S. Dimitrijević ${ }^{2}$ (D) and Zoran Simić ${ }^{2}$ \\ 1 Laboratoire PIIM, Aix-Marseille Université, CNRS, 13397 Marseille Cedex 20, France; \\ missny0909@gmail.com (N.K.); ibtissem.hannachi@yahoo.fr (I.H.); mohammed.koubiti@univ-amu.fr (M.K.); \\ yannick.marandet@univ-amu.fr (Y.M.); roland.stamm@univ-amu.fr (R.S.) \\ 2 Astronomical Observatory, Volgina 7, 11060 Belgrade 38, Serbia; mdimitrijevic@aob.rs (M.S.D.); \\ zsimic@aob.rs (Z.S.) \\ * Correspondence: joel.rosato@univ-amu.fr; Tel.: +33-491-288-624
}

Academic Editor: Ulrich D. Jentschura

Received: 23 August 2017; Accepted: 29 September 2017; Published: 4 October 2017

\begin{abstract}
The shape of atomic spectral lines in plasmas contains information on the plasma parameters, and can be used as a diagnostic tool. Under specific conditions, the plasma located at the edge of tokamaks has parameters similar to those in magnetic white dwarf stellar atmospheres, which suggests that the same line shape models can be used. A problem common to tokamak and magnetic white dwarfs concerns the modeling of Stark broadening of hydrogen lines in the presence of an external magnetic field and the related Zeeman effect. In this work, we focus on a selection of issues relevant to Stark broadening in magnetized hydrogen plasmas. Various line shape models are presented and discussed through applications to ideal cases.
\end{abstract}

Keywords: line shapes; Stark broadening; Zeeman effect; tokamaks; white dwarfs

\section{Introduction}

Tokamaks are devices of toroidal shape employed in controlled nuclear fusion research for the confinement of a burning hydrogen plasma, with ion and electron temperatures in the order of $10^{8} \mathrm{~K}$. The ITER project ( $w w w . i t e r . o r g$ ) aims to demonstrate the principle of producing more energy from the fusion process than is used to initiate it; this large-scale tokamak is presently under construction in France, and it should be operational for first plasma discharge by the end of 2025 [1]. In order to support the operation of the machine, an extensive set of spectroscopic measurements is planned [2]; for example, passive spectroscopy of the Balmer series will provide information on the isotopic proportion of the fueling gas $\left(N_{T} / N_{D}, N_{H} / N_{D}\right)$ in the divertor region [3], where a large amount of neutrals is expected. Under specific conditions (plasma in "detached" regime), the electron density in the divertor can attain values larger than $10^{14} \mathrm{~cm}^{-3}$, so that the Stark effect due to the plasma microfield becomes the dominant line-broadening mechanism on lines with a high principal quantum number. Recent observations of $\mathrm{D} \varepsilon$ (transition $7 \rightarrow 2$ of deuterium) in the divertor of ASDEX-Upgrade have been used for the determination of the electron density, using the Stark broadening of the line [4]. Balmer lines with a low principal quantum number like $\mathrm{D} \alpha$ are also affected by the microfield and Stark broadening enters into competition with both Doppler broadening and Zeeman splitting [5]. The need for accuracy in spectroscopic diagnostics of tokamak edge plasmas has prompted an interest in the development of line broadening models accounting for the simultaneous action of electric and magnetic fields on atomic energy levels, e.g., [6]. Recently, a line shape database for the first Balmer lines 
accounting for the Stark and Zeeman effects has been devised for tokamak spectroscopy applications using computer simulations [7]. Similar databases for Stark broadening exist in astrophysics for stellar atmosphere diagnostic applications, e.g., STARK-B (stark-b.obspm.fr) [8]. Magnetic white dwarfs are of particular interest because they present Balmer spectra with Zeeman triplets $[9,10]$ just as in tokamaks, and atmosphere models indicate zones where the electron density value is comparable to that in detached divertor plasmas [11]. Within this context, modeling work by tokamak spectroscopists in conjunction with astrophysicists aimed at improving Stark line shape databases is presently ongoing. In this paper, we report on models and techniques used for the description of Stark-Zeeman line shapes. A special emphasis is put on ion dynamics and collision operator models.

\section{Stark Broadening Formalism}

The formalism used in Stark line shape modeling involves atomic physics and statistical physics. An atom immersed in a plasma and emitting a photon is considered; during photon emission, the energy levels are perturbed due to the presence of the charged particles surrounding the atom, and this perturbation results in a splitting and broadening of spectral lines. The Doppler effect, which stems from the atom's motion, is not considered, hereafter, for the sake of clarity (it can be accounted for through convolution). According to standard textbooks (e.g., [12]), a line shape $I(\omega)$ is given by the Fourier transform of the atomic dipole autocorrelation function, a quantity described in the framework of quantum mechanics

$$
\begin{gathered}
I(\omega)=\frac{1}{\pi} \operatorname{Re} \int_{0}^{\infty} d t C(t) e^{i \omega t} \\
C(t)=\sum_{\alpha \alpha \prime \beta \beta / \varepsilon} \rho_{\alpha \alpha}\left(\mathbf{d}_{\alpha \beta} \cdot \varepsilon\right)\left(\mathbf{d}_{\alpha \prime \beta \prime}^{*} \cdot \varepsilon\right)\left\{U_{\alpha \prime \alpha}(t) U_{\beta \prime \beta}^{*}(t)\right\}
\end{gathered}
$$

Here, $\rho$ is the restriction of the density operator to the atomic Hilbert space evaluated at initial time, $\mathbf{d} \cdot \varepsilon$ is the dipole projected onto the polarization vector $\varepsilon$, the indices $\alpha, \alpha^{\prime}$ (resp. $\beta, \beta^{\prime}$ ) are used for matrix elements and denote states in the upper (resp. lower) level, the brackets $\{\ldots\}$ denote an average over the perturber trajectories, and $U(t)$ is the evolution operator. It obeys the time-dependent Schrödinger equation

$$
i \hbar \frac{d U}{d t}(t)=\left[H_{0}+V(t)\right] U(t)
$$

Here $H_{0}$ is the Hamiltonian accounting for the atomic energy level structure and $V(t)=-\mathbf{d} . \mathbf{F}(t)$ is the time-dependent Stark effect term resulting from the action of the microscopic electric field $\mathbf{F}(t)$ and written in the Schrödinger picture, here. When this term is neglected, the Schrödinger equation has the trivial solution $U(t)=\exp \left(-i H_{0} t / \hbar\right)$, which shows, using Equations (2) and (1), that $I(\omega)$ reduces to a set of delta functions (or Lorentzian functions if the natural broadening is retained). By contrast, the case where $\mathbf{F}(t)$ is significant is much trickier, because there is no general exact analytical solution. The time-dependent perturbation theory yields a formal expansion (Dyson series), which is not applicable in explicit calculations because of the non-commutation of the interaction term at different times (time-ordering problem). This concerns, in particular, the microfield due to ions. Several models, based on suitable approximations, have been developed in such a way to provide an analytical expression for the line shape (e.g., the impact and static approximations, the model microfield method, etc.). On the other hand, computer simulations involving the numerical integration of the Schrödinger Equation (3) [13,14] provide reference line profiles and can serve as a benchmark for testing models. In the following, we focus on the ion dynamics issue, which is important for hydrogen lines with a low principal quantum number.

\section{Ion Broadening at the Impact Limit}

Modeling work has been done over the last decade, in order to set up an analytical formula for the Lyman $\alpha$ and Lyman $\beta$ line shapes for opacity investigations in tokamak divertor plasmas ([15] and Refs. therein). Kinetic simulations of radiation transport using the EIRENE code (www.eirene.de) have 
indicated the possibility of an alteration of the ionization-recombination balance due to the Lyman opacity [16]. The code employs a Monte Carlo method with random walks and related estimators; line shapes in the atomic frame of reference, i.e., without Doppler broadening, are involved in the sampling of photon frequencies. Because of the low principal quantum numbers $(n=2$ and 3$)$ involved in Lyman $\alpha$ and Lyman $\beta$, the ion dynamics is important, and a collision operator can be used for ions as a first approach for an estimate of the Stark broadening. The model of Griem, Kolb, and Shen (GKS, [17]), developed for electron broadening in hydrogen plasmas, can be adapted in a simple way for ions. Consider a single ion perturbing the emitter (binary interaction); the collision operator $K$ reads

$$
K=N \int_{0}^{\infty} d v f(v) v \int_{0}^{\lambda_{D}} d b 2 \pi b\{1-S\}_{\text {angle }}
$$

where the integrals are done over the velocity (module) $v$ and the impact parameter (module) $b, N$ and $f$ stand for the perturber's density and velocity distribution function, the brackets denote an angular average and $S$ is the scattering matrix corresponding to a binary collision. The emitter motion is accounted for through a reduced mass model. The upper bound $\lambda_{D}$ is the Debye length, which accounts phenomenologically for the screening of the Coulomb field due to the electrons. In the GKS model, the integration domain is restricted in such a way that $S$ can be expanded at the second order in the Stark perturbation $V(t)=-\mathbf{d} . \mathbf{F}(t)$ ("weak collisions"); the integrals can be performed explicitly in the case of a Coulomb field and the resulting collision operator reads (notations are different from those in [17])

$$
K_{\text {weak }}=N \pi b_{W}^{2} v_{0} \times \frac{2}{\sqrt{\pi}} \frac{r^{2}}{n^{4}} E_{1}\left[\left(\frac{b_{W}}{\lambda_{D}}\right)^{2} \times \frac{r^{2}}{n^{4}}\right]
$$

Here, $v_{0}=\sqrt{ }\left(2 k_{B} T / m_{\text {red }}\right)$ is the thermal velocity, accounting for reduced mass; $b_{W}=(2 / 3)^{1 / 2} \hbar n^{2} / m_{e} v_{0}$ is the Weisskopf radius, which discriminates between weak $\left(b>b_{W}\right)$ and strong $\left(b<b_{W}\right)$ collisions (sometimes this is defined without the $(2 / 3)^{1 / 2}$ factor); $r^{2}$ is the square of the atomic electron position operator, in atomic units, restricted to the subspace relative to quantum number $n$, and can be formally replaced by its diagonal matrix element $(9 / 4) n^{2}\left(n^{2}-l^{2}-l-1\right)$; and $E_{1}$ is the exponential integral function. Equation (5) represents the contribution of weak collisions only; these are usually dominant with respect to strong collisions, that the impact approximation is valid. An estimate of the strong collision contribution to $K$ can be done by setting formally $S \equiv 0$ in Equation (4) (this function strongly oscillates with respect to $b$ and $v$ ) and adjusting the integration domain in such a way that only impact parameters smaller than the Weisskopf radius are retained. An explicit calculation yields

$$
K_{\text {strong }}=N \pi b_{W}^{2} v_{0} \times \frac{2}{\sqrt{\pi}} \frac{r^{2}}{n^{4}}
$$

and the final expression for the collision operator is $K=K_{\text {weak }}+K_{\text {strong. }}$. A Stark line shape described within the impact approximation can be written as a sum of Lorentzian functions with a half-width at half-maximum given by the matrix elements of the collision operator. In the framework of radiative transfer Monte Carlo simulations, this renders the sampling of photon frequencies straightforward. Figure 1 shows a plot of Lyman $\alpha$ at conditions of tokamak edge plasmas, calculated without Doppler broadening and using the GKS collision operator. The result is in contrasted to the result of a computer simulation, where the Schrödinger equation is solved numerically for a given set of microfield histories. These histories are simulated using particles moving in a cube with periodic boundary conditions (see [7] for details on the code). As can be seen, the impact approximation agrees well with the simulation result. Two essential features of the impact model are the assumptions of binary collision and short collision time; these features are characterized by the ratios $b_{W} / r_{0}$ and $\tau_{c} / t_{i}$, which must be much smaller than unity. Here, $r_{0} \sim N^{-1 / 3}$ is the mean interparticle distance, $\tau_{c} \sim r_{0} / v_{0}$ is the collision time, and $t_{i}$ is the time of interest (which is estimated as the inverse line width). Here, the values of 
$3 \times 10^{12} \mathrm{~cm}^{-3}$ and $1 \mathrm{eV}$, assumed for density and temperature, respectively, yield values of $4 \%$ and $0.6 \%$ for these ratios.

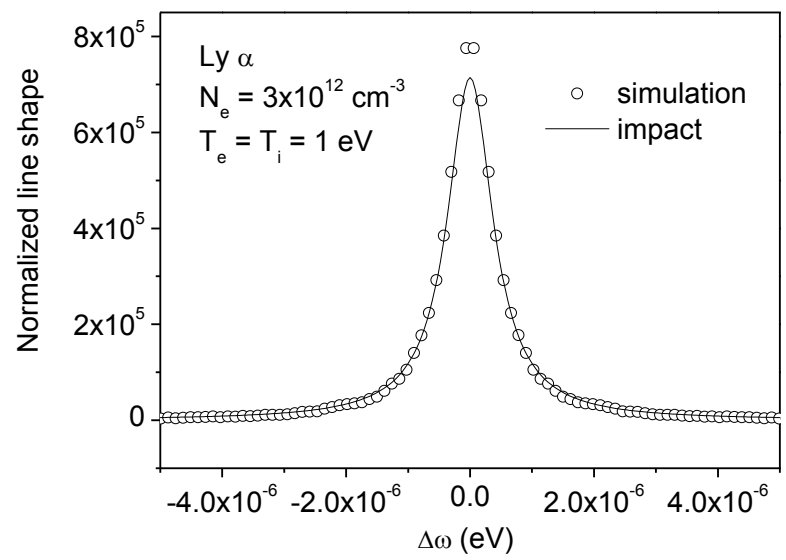

Figure 1. Deuterium Lyman $\alpha$ line profile in the atomic frame of reference, i.e., without Doppler broadening, in conditions such that the ratios $b_{W} / r_{0}$ and $\tau_{c} / t_{i}$ are much smaller than unity (see text). As can be seen, the impact theory agrees well with the simulation.

\section{Influence of Zeeman Effect on Line Broadening}

In tokamaks, the magnetic field is strong enough that hydrogen lines exhibit a Zeeman triplet structure. This structure is a feature of energy degeneracy removal relative to the magnetic quantum number $m$; it can alter the Stark broadening if the Zeeman perturbation $\hbar \omega_{Z}=\left|\mu_{B} B\right|$ ( $\mu_{B}$ being the Bohr magneton) exceeds the characteristic Stark perturbation, estimated either by $n^{2} e a_{0} F_{0}$ (static picture, $F_{0}$ being the Holtsmark field) or $\hbar N \pi b_{W}{ }^{2} v_{0} \ln \left(\lambda_{D} / b_{W}\right)$ (impact limit, the logarithm stems from the behavior of the exponential integral for small argument). An extension of the GKS impact collision operator that accounts for this degeneracy removal can be devised through the use of an alternative $S$ operator in Equation (4) involving the Zeeman Hamiltonian. The method employs an adaptation of the GBKO model (Griem, Baranger, Kolb, Oertel [18]), initially developed for helium lines, for hydrogen lines with Zeeman effect; details can be found in $[6,19]$. The result is a collision operator with a dependence on the $m$ quantum number. The following relation holds

$$
\begin{gathered}
\langle\alpha|K| \alpha\rangle=N \int_{0}^{\infty} d v f(v) v \sigma_{\alpha}(v) \\
\sigma_{\alpha}=\pi\left(b_{\alpha}^{\text {st }}\right)^{2}+2 \pi b_{W \alpha}^{2}\left\{K_{\alpha}^{\prime /} \ln \left(\frac{\lambda_{D}}{b_{\alpha}^{\text {st }}}\right)+\left[K_{\alpha}^{\perp} a(s)-i K_{\alpha}^{\prime \perp} b(s)\right]_{s=\lambda_{D} / b_{m}}^{s=b_{\alpha}^{\text {st }} / b_{m}}\right\}
\end{gathered}
$$

where $\alpha$ stands for the quantum numbers $n, l, m$; the constants $K_{\alpha} / /=\Sigma_{\alpha^{\prime}}\left|z_{\alpha^{\prime} \alpha}\right|^{2} / n_{\alpha} 4$, $K_{\alpha}{ }^{\perp}=\Sigma_{\alpha^{\prime}}\left[\left|x_{\alpha^{\prime} \alpha}{ }^{+}\right|^{2}+\left|x_{\alpha^{\prime} \alpha}{ }^{-1}\right|^{2}\right] / n_{\alpha}{ }^{4}$, and $K_{\alpha}^{\prime}{ }^{\perp}=\Sigma_{\alpha^{\prime}}\left[\left|x_{\alpha^{\prime} \alpha}{ }^{+}\right|^{2}-\left|x_{\alpha^{\prime} \alpha}{ }^{-}\right|^{2}\right] / n_{\alpha}{ }^{4}$ involve the atomic electron position operator (here, by convention, $\left.x^{ \pm}=(x \pm i y) / \sqrt{ } 2\right) ; a(s)$ and $b(s)$ are the GBKO functions writable in terms of the modified Bessel functions, $b_{\alpha}{ }^{\text {st }}$ is the strong collision radius; and $b_{m}=v / \omega_{Z}$ is a distance relative to Zeeman degeneracy removal. The latter mitigates the characteristic strength of the Stark perturbation. If the ordering $b_{W \alpha}<<b_{m}$ is satisfied, the $a$ function evaluated at the upper bound can be replaced by a logarithm, and the length $b_{m}$ plays a role similar to the Debye length. Note that the Weisskopf radius here is a function of $v$; the definition given in the previous section corresponds to evaluation at the thermal velocity $v=v_{0}$. In Equation (8), the dependence on velocity has not been written explicitly for the sake of simplicity. The so-called "strong collision radius" - here, $b_{\alpha}{ }^{\text {st }}$-coincides with the Weisskopf radius at the limit $\mathrm{B} \rightarrow 0$, and is different at finite magnetic field. It can be determined by the solving of an integral equation; see $[6,19]$ for details. The mitigation of Stark broadening due to the Zeeman effect is all the more important so that the Zeeman energy 
sublevels are separated. This effect is particularly important on the lateral components of Lyman $\alpha$ because they are not affected by $\Delta m=0$ couplings (i.e., $K_{\alpha} / /$ vanishes identically in Equation (8)). Figure 2 shows an illustration of the Stark broadening mitigation on the lateral blue component. The same plasma conditions as in the previous section are considered, and a magnetic field of $2 \mathrm{~T}$ is assumed. The adaptation of the GBKO model yields a reduction of the line width by a factor of three. A slight shift towards the blue side is also visible; this corresponds to the imaginary part of the collision operator matrix element, and its amplitude is determined by the GBKO $b$ function.

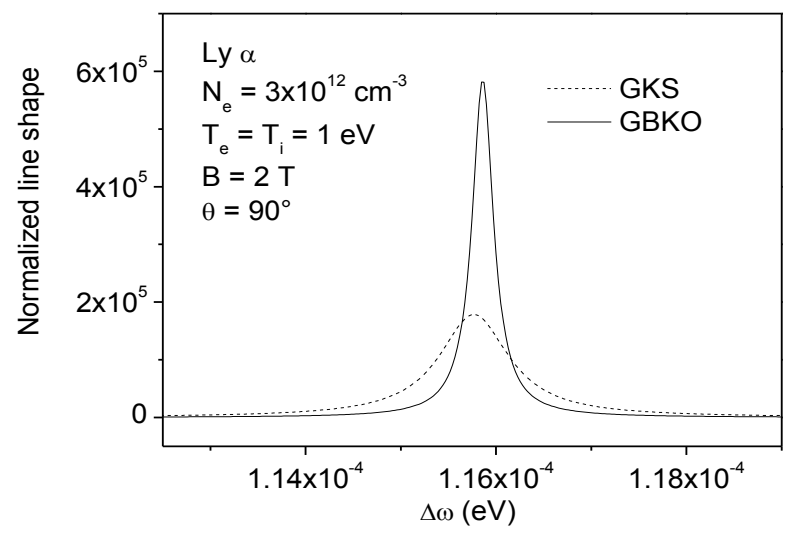

Figure 2. Lateral blue component of the Lyman $\alpha$ Zeeman triplet in the atomic frame of reference. A magnetic field of $2 \mathrm{~T}$ is assumed and the observation is assumed perpendicular to $B\left(\theta=90^{\circ}\right)$. "GBKO" refers to the Griem-Baranger-Kolb-Oertel 1962 model, adapted to hydrogen in the presence of Zeeman effect; "GKS" refers to the original Griem-Kolb-Shen 1959 model for hydrogen without degeneracy removal. As can be seen, the Zeeman degeneracy removal, retained in the GBKO model, strongly reduces the line width. A slight shift is also visible.

\section{An Extension of the Impact theory to Non-Binary Interactions}

The impact approximation for ions can be inaccurate under high density or low temperature conditions, namely, when the dimensionless parameters $\langle\alpha|K| \alpha\rangle \times \tau_{\mathcal{c}}$ and $b_{W} / r_{0}$ are significant. Comparisons with ab initio simulations have indicated that the use of the impact approximation can result in a systematic overestimate of the Stark width, e.g., [6]. Recently, a model has been developed in such a way to account for the deviations analytically [20]. The model uses kinetic theory techniques and is inspired by the "unified theory", a formalism proposed in the late sixties as an extension of the impact approximation for electrons [21,22]. The main result of the unified theory is a frequency-dependent collision operator, which accounts for incomplete collisions (i.e., collisions not achieved during the time of interest) and provides a correct asymptotic line shape formula. If we apply the unified theory to ions, the collision operator reads

$$
K(\omega)=N \int_{0}^{\infty} d t e^{i \omega t}\left\{V(t) e^{-i H_{0} t / \hbar} Q(t) V(0)\right\}
$$

where $V$ stands for the Stark interaction corresponding to a binary collision with an ion, $\mathrm{N}$ is the number of ions, the brackets $\{\ldots\}$ denote statistical average, and $Q(t)$ is the evolution operator of the atom under the influence of one collision in the interaction picture. It obeys the Schrödinger equation and admits the Dyson series as a formal expression. Several techniques exist for the evaluation of the integral, either based on the exact solution or on the perturbation theory, as done for the impact collision operator. The behavior of the collision operator at small and large frequency detuning is obtained by asymptotic analysis. This shows that $K(\omega)$ behaves as $|\Delta \omega|^{-1 / 2}$ in the wings, and reduces to the impact collision operator at the center. For applications in tokamak edge and divertor conditions, it has been necessary to modify the original model Equation (9) in such a way as to account for $\mathrm{N}$ body 
interaction effects, referred to as "correlated collisions". Such correlations occur if, during one collision, the atom "feels" the presence of the other perturbers. Correlated collisions are important when the characteristic collision frequency $\langle\alpha|K| \alpha>$ becomes of the same order as, or larger than, the inverse correlation time of the emitter-perturber interaction potential $[23,24]$. The latter is of the order of the inverse plasma frequency $\omega_{p i}{ }^{-1}$ (due to Debye screening). In the model reported in [20], correlated collisions are retained through a resummation procedure applied to kinetic equations of BBGKY-type. The resulting collision operator obeys a nonlinear equation with a structure similar to Equation (9)

$$
K(\omega)=N \int_{0}^{\infty} d t e^{i \omega t}\left\{V(t) e^{-i\left(H_{0}-i K_{0}\right) t / \hbar} Q(t) V(0)\right\}
$$

In the exponential, $K_{0}=K\left(\omega_{0}\right)$ is the collision operator evaluated at the central frequency of the line under consideration. Its presence denotes a non-Hermitian part in the atomic Liouvillian, which can be interpreted as a renormalization or "dressing" of the atomic energy levels, induced by the presence of other perturbers during a single collision and their correlation with the collision under consideration. This model presents similarities to the result of the resonance broadening theory used for plasma turbulence $[25,26]$. In practice, a calculation of the collision operator from Equation (10) should be done by iterations. Tests have indicated that such a procedure yields a fast convergence, with typically no more than ten iterations. Figure 3 presents a plot of Lyman $\alpha$ broadened due to ions at $N_{e}=10^{15} \mathrm{~cm}^{-3}, T_{e}=T_{i}=1 \mathrm{eV}$, which was obtained using impact approximation, the unified theory Equation (9), and its extension Equation (10) applied to ions. No magnetic field is retained here. A numerical result from a simulation is also shown in the figure. As can be seen, the impact approximation strongly overestimates the width. This stems from the inadequacy of this model as a regime where incomplete and correlated collisions are present. The extended unified theory gives a much better result, with an overestimate of the width no larger than $20 \%$. We can also see that the standard unified theory (i.e., not accounting for correlated collisions) is not sufficient here, and yields a different (and incorrect) shape structure, with a dip at the center. Correlated collisions are important in this region, because they govern the average atomic evolution operator at long times; hence, by virtue of the Fourier transform, at small frequency detuning. The typical range for the dip corresponds to frequencies smaller than the matrix elements of $K_{0}$.

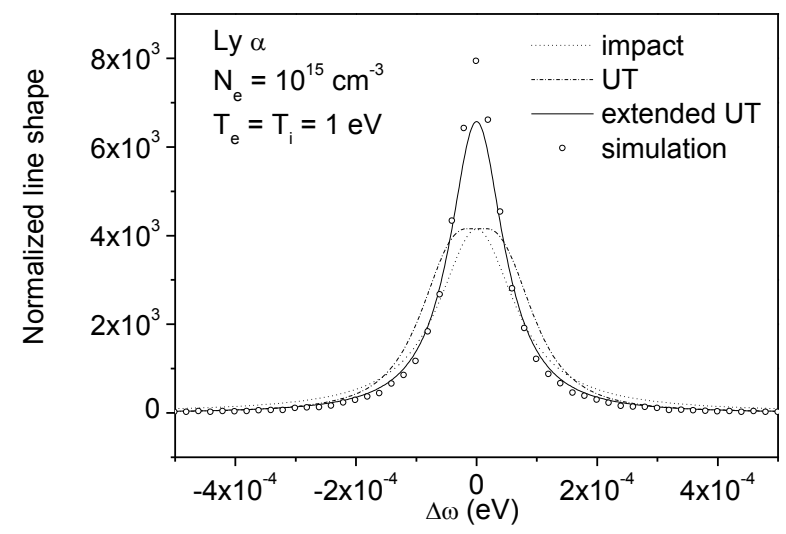

Figure 3. Profile of the Lyman $\alpha$ line at conditions such that neither the impact approximation nor the unified theory (UT) for ions are valid. The extension of the unified theory (UT), which accounts for correlated collisions, provides a good estimate of the line width.

\section{Stark-Zeeman Line Shapes in Magnetic White Dwarfs}

About $10 \%$ of white dwarfs are known to have a magnetic field strength of $10^{5}$ to $5 \times 10^{8} \mathrm{G}$ $\left(10\right.$ to $\left.5 \times 10^{4} \mathrm{~T}\right)$, as indicated by spectroscopic observations and models [9,10,27]. An interpretation of the shape of absorption lines requires the Zeeman effect be accounted for in line broadening 
models, as done in tokamak edge plasma spectroscopy. Work is currently ongoing in order to improve already-existing databases by implementing the Zeeman effect in Stark line shape models. Figure 4 shows an illustration of the importance of the Zeeman effect in white dwarf spectra. A profile of $\mathrm{H} \alpha$ has been calculated with a numerical simulation assuming $N_{e}=10^{17} \mathrm{~cm}^{-3}, T_{e}=T_{i}=0.5 \mathrm{eV}, B=500 \mathrm{~T}$ and observation perpendicular to $B$. These conditions are characteristic of the atmosphere of a white dwarf of DAH (magnetic DA) type, presenting hydrogen absorption lines. The spectrum exhibits a triplet structure characteristic to the Zeeman effect. The description of Stark broadening can be more challenging than in tokamak devices because particle correlations are important (here $r_{0} / \lambda_{D}$ is of the order of unity) and the quasiparticle model used in the simulation may not be accurate enough. An extension of this work would consist of using full molecular dynamic simulations. Features specific to dense plasmas, such as quadrupole interactions, could also be important, and should be addressed in detail; see [28] for recent works. The presence of a strong magnetic field changes the plasma dynamics through the gyromotion effect and this can result in an alteration of electron collision operator formulas $[29,30]$. Specific modifications to the atomic energy level structure (quadratic Zeeman effect, Lorentz electric field) could also be important [31].

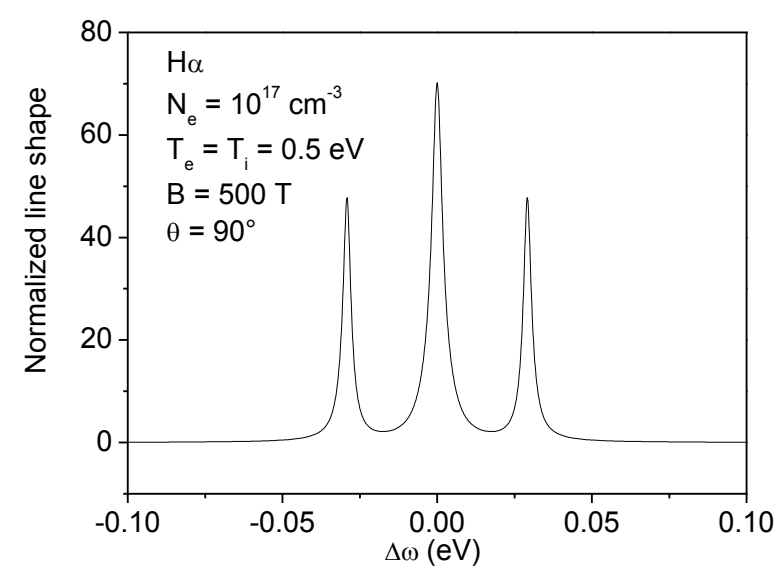

Figure 4. Balmer $\alpha$ line shape in conditions relevant to DAH white dwarf atmospheres. The spectrum exhibits a triplet structure due to the strong magnetic field.

\section{Non-Hydrogen Species}

Tokamak plasmas contain a sufficient amount of impurities, due to plasma-wall interactions or vacuum chamber conditioning, to be observable on spectra (e.g., oxygen, nitrogen, carbon [32]). Specific atomic species of high Z can be found in some, but not all, tokamaks (e.g., tungsten [33]). Helium is also expected to be present in high amounts in ITER during the nuclear phase, where discharges with tritium will be done. As a rule, an analysis of the position and the intensity of impurity lines provides information on the plasma composition, the recycling and sputtering processes, which helps in the setting up of tokamak discharge scenarios. The design of fast routines employs line shape codes (e.g., PPP [34]), and can also use databases available in astrophysics such as STARK-B (stark-b.obspm.fr). An issue that is specific to non-hydrogen lines concerns the modeling of collisional electron broadening, accounting for degeneracy removal with quantum number $l$. This degeneracy removal yields a reduction of the Stark effect and can induce a shift on the line under consideration. An illustration of this effect is shown in Figure 5. The He $492 \mathrm{~nm}$ line $\left(1 \mathrm{~s} 4 \mathrm{~d}{ }^{1} \mathrm{D}-1 \mathrm{~s} 2 \mathrm{p}{ }^{1} \mathrm{P}\right)$ has been addressed, assuming $N_{e}=10^{15} \mathrm{~cm}^{-3}$ and $T_{e}=10,000 \mathrm{~K}$, without ion broadening and without magnetic field for the sake of clarity. Two Lorentzian curves are displayed in the graph. One corresponds to the result of a hydrogen approximation (through the GKS impact model discussed above), and the other one corresponds to the model reported in the STARK-B database, which accounts for the degeneracy removal. As can be seen, the line width is sensitive to the model used. The shift visible in the STARK-B 
result is a feature of the degeneracy removal. It depends on the plasma parameters, and can also be used for diagnostic purposes. An extension of already-existing databases to magnetized plasma conditions, where the Zeeman effect is visible in spectra, is under consideration. Work is presently ongoing for applications both in magnetic fusion and astrophysical plasmas.

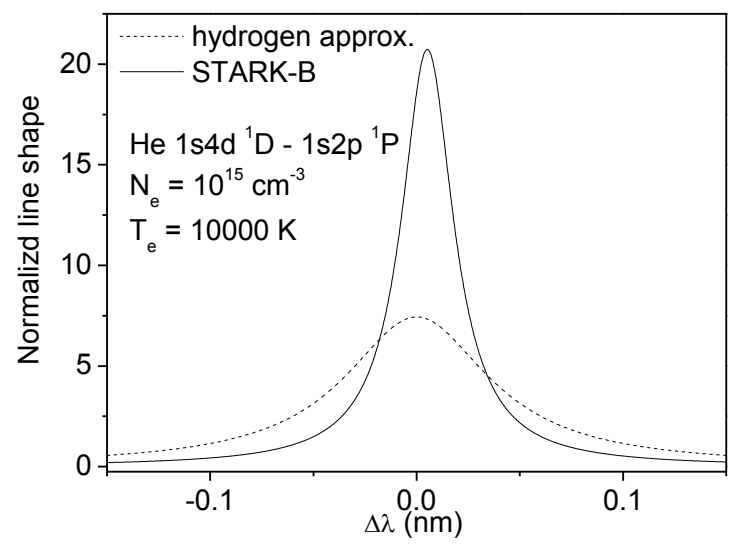

Figure 5. Plot of the electron impact broadening of He $1 \mathrm{~s} 4 \mathrm{~d}^{1} \mathrm{D}-1 \mathrm{~s} 2 \mathrm{p}{ }^{1} \mathrm{P}$ calculated using the STARK-B database (solid line) and a hydrogen approximation for the energy level structure (dashed line). Conditions relevant to high-density tokamak divertor plasmas are considered here, and no magnetic field is retained for the sake of clarity. As can be seen, the result significantly depends on the model used.

\section{Conclusions}

The shape of atomic spectral lines in plasmas contains information on the plasma parameters, and can be used as a diagnostic tool. In this work, we have examined a selection of problems involved in the modeling of Stark broadening for applications in magnetic fusion and in astrophysics. A specific issue concerns the description of ion dynamics on hydrogen lines, which is important if the upper principal quantum number is low as on Balmer $\alpha$. The use of a collision operator for ions is convenient because the line shape can be described analytically. In this framework, we have shown that the early model by Griem et al. (GBKO) for atoms with nondegenerate levels can be adapted to hydrogen in the presence of the Zeeman effect. It coincides with numerical simulations at the so-called impact limit, i.e., when the microfield fluctuates at a time scale much smaller than the time of interest, and when strong collisions are rare events. We have also shown that deviations in the impact limit due to non-binary interactions can be retained through an adaptation of the so-called unified theory, a formalism based on kinetic equations and related statistical concepts (BBGKY hierarchy). In astrophysics, magnetic white dwarfs present similarities with tokamak edge plasmas because the hydrogen absorption lines in atmospheres can exhibit a Zeeman triplet structure. We have illustrated this point through calculation of an absorption line in conditions relevant to a DAH stellar atmosphere. In this framework, an extension of already-existing Stark databases that is devoted to accounting for the Zeeman effect is presently under preparation. We have also shown that the description of the broadening of non-hydrogen lines is a challenging issue, both in astrophysics and in magnetic fusion. Modeling work is also ongoing in this framework in order to improve the databases.

Acknowledgments: This work is supported by the funding agency Campus France (Pavle Savić PHC project 36237PE). This work has also been carried out within the framework of the EUROfusion Consortium and has received funding from the Euratom research and training programme 2014-2018 under Grant agreement no 633053. The views and opinions expressed herein do not necessarily reflect those of the European Commission.

Author Contributions: All authors contributed equally to this work.

Conflicts of Interest: The authors declare no conflict of interest. 


\section{References}

1. ITER Press Release. 16 June 2016. Available online: https://www.iter.org/news/pressreleases (accessed on 3 July 2017).

2. Donne, A.J.H.; Costley, A.E.; Barnsley, R.; Bindslev, H.; Boivin, R.; Conway, G.; Fisher, R.; Giannella, R.; Hartfuss, H.; von Hellermann, M.G.; et al. Progress in the ITER Physics Basis, Chapter 7: Diagnostics. Nucl. Fusion 2007, 47, S337-S384. [CrossRef]

3. Sugie, T.; Costley, A.; Malaquias, A.; Walker, C. Spectroscopic Diagnostics for ITER. J. Plasma Fusion Res. 2003, 79, 1051-1061. [CrossRef]

4. Potzel, S.; Dux, R.; Müller, H.W.; Scarabosio, A.; Wischmeier, M.; ASDEX Upgrade Team. Electron density determination in the divertor volume of ASDEX Upgrade via Stark broadening of the Balmer lines. Plasma Phys. Control. Fusion 2014, 56, 025010. [CrossRef]

5. Rosato, J.; Kotov, V.; Reiter, D. Modelling of passive spectroscopy in the ITER divertor: The first hydrogen Balmer lines. J. Phys. B At. Mol. Opt. Phys. 2010, 43, 144024. [CrossRef]

6. Rosato, J.; Marandet, Y.; Capes, H.; Ferri, S.; Mossé, C.; Godbert-Mouret, L.; Koubiti, M.; Stamm, R. Stark broadening of hydrogen lines in low-density magnetized plasmas. Phys. Rev. E 2009, 79, 046408. [CrossRef] [PubMed]

7. Rosato, J.; Marandet, Y.; Stamm, R. A new table of Balmer line shapes for the diagnostic of magnetic fusion plasmas. J. Quant. Spectrosc. Radiat. Transf. 2017, 187, 333-337. [CrossRef]

8. Sahal-Bréchot, S.; Dimitrijević, M.S.; Moreau, N.; Ben Nessib, N. The STARK-B database VAMDC node: A repository for spectral line broadening and shifts due to collisions with charged particles. Phys. Scr. 2015, 90, 054008. [CrossRef]

9. Kepler, S.O.; Pelisoli, I.; Jordan, S.; Kleinman, S.J.; Kulebi, B.; Koester, D.; Peçanha, V.; Castanheira, B.G.; Nitta, A.; da Silveira Costa, J.E.; et al. Magnetic white dwarf stars in the Sloan Digital Sky Survey. Mon. Not. R. Astron. Soc. 2013, 429, 2934-2944. [CrossRef]

10. Külebi, B.; Jordan, S.; Euchner, F.; Gänsicke, B.T.; Hirsch, H. Analysis of hydrogen-rich magnetic white dwarfs detected in the Sloan Digital Sky Survey. Astron. Astrophys. 2009, 506, 1341-1350. [CrossRef]

11. Wickramasinghe, D.T. Model atmospheres for DA and DB white dwarfs. Mon. Not. R. Astron. Soc. 1972, 76, 129-179.

12. Griem, H.R. Spectral Line Broadening by Plasmas; Academic Press: London, UK, 1974.

13. Stamm, R.; Smith, E.W.; Talin, B. Study of hydrogen Stark profiles by means of computer simulation. Phys. Rev. A 1984, 30, 2039-2046. [CrossRef]

14. Stambulchik, E.; Maron, Y. Plasma line broadening and computer simulations: A mini-review. High Energy Density Phys. 2010, 6, 9-14. [CrossRef]

15. Rosato, J.; Reiter, D.; Kotov, V.; Marandet, Y.; Capes, H.; Godbert-Mouret, L.; Koubiti, M.; Stamm, R. Progress on Radiative Transfer Modelling in Optically Thick Divertor Plasmas. Contrib. Plasma Phys. 2010, 50, $398-403$. [CrossRef]

16. Kotov, V.; Reiter, D.; Kukushkin, A.S.; Pacher, H.D.; Börner, P.; Wiesen, S. Radiation Absorption Effects in B2-EIRENE Divertor Modelling. Contrib. Plasma Phys. 2006, 46, 635-642. [CrossRef]

17. Griem, H.R.; Kolb, A.C.; Shen, K.Y. Stark Broadening of Hydrogen Lines in a Plasma. Phys. Rev. 1959, 116, 4-16. [CrossRef]

18. Griem, H.R.; Baranger, M.; Kolb, A.C.; Oertel, G. Stark Broadening of Neutral Helium Lines in a Plasma. Phys. Rev. 1962, 125, 177-195. [CrossRef]

19. Rosato, J.; Capes, H.; Godbert-Mouret, L.; Koubiti, M.; Marandet, Y.; Stamm, R. Accuracy of impact broadening models in low-density magnetized hydrogen plasmas. J. Phys. B At. Mol. Opt. Phys. 2012, 45, 1-7. [CrossRef]

20. Rosato, J.; Capes, H.; Stamm, R. Influence of correlated collisions on Stark-broadened lines in plasmas. Phys. Rev. E 2012, 86, 046407. [CrossRef] [PubMed]

21. Voslamber, D. Unified Model for Stark Broadening. Z. Naturforsch. 1969, 24, 1458-1472.

22. Smith, E.W.; Cooper, J.; Vidal, C.R. Unified Classical-Path Treatment of Stark Broadening in Plasmas. Phys. Rev. 1969, 185, 140-151. [CrossRef]

23. Capes, H.; Voslamber, D. Electron Correlations in the Unified Model for Stark Broadening. Phys. Rev. A 1972, 5, 2528-2536. [CrossRef] 
24. Capes, H.; Voslamber, D. Spectral-line profiles in weakly turbulent plasmas. Phys. Rev. A 1977, 15, 1751-1766. [CrossRef]

25. Dupree, T.H. A Perturbation Theory for Strong Plasma Turbulence. Phys. Fluids 1966, 9, 1773-1782. [CrossRef]

26. Weinstock, J. Formulation of a Statistical Theory of Strong Plasma Turbulence. Phys. Fluids 1969, 12, 1045-1058. [CrossRef]

27. Landstreet, J.D.; Bagnulo, S.; Valyavin, G.G.; Fossati, L.; Jordan, S.; Monin, D.; Wade, G.A. On the incidence of weak magnetic fields in DA white dwarfs. Astron. Astrophys. 2012, 545, A30. [CrossRef]

28. Gomez, T.A.; Nagayama, T.; Kilcrease, D.P.; Montgomery, M.H.; Winget, D.E. Effect of higher-order multipole moments on the Stark line shape. Phys. Rev. A 2016, 94, 022501. [CrossRef]

29. Maschke, E.K.; Voslamber, D. Stark broadening of hydrogen lines in strong magnetic fields. In Proceedings of the Seventh International Conference, Beograd, Yugoslavia, 22-27 August 1965; Gradevinska Knjiga Publishing House : Beograd, Yugoslavia, 1966; Volume II, p. 568.

30. Rosato, J. Cases 10-11: B-induced trajectory effects. In Proceedings of the 4th Spectral Line Shapes in Plasmas (SLSP) Code Comparison Workshop, Vienna, Austria, 20-24 March 2017.

31. Kieu, N.; Rosato, J.; Stamm, R.; Kovacević-Dojcinović, J.; Dimitrijević, M.S.; Popović, L.C.; Simić, Z. A New Analysis of Stark and Zeeman Effects on Hydrogen Lines in Magnetized DA White Dwarfs. Atoms 2017, submitted.

32. Koubiti, M.; Nakano, T.; Capes, H.; Marandet, M.; Mekkaoui, A.; Mouret, L.; Rosato, J.; Stamm, R. Characterization of the JT-60U Divertor Plasma Region During the Formation of a Strong Radiation. Contrib. Plasma Phys. 2012, 52, 455-459. [CrossRef]

33. Pütterich, T.; Neu, R.; Dux, R.; Whiteford, A.D.; O'Mullane, M.G. The ASDEX Upgrade Team. Modelling of measured tungsten spectra from ASDEX Upgrade and predictions for ITER. Plasma Phys. Control. Fusion 2008, 50, 085016. [CrossRef]

34. Calisti, A.; Mossé, C.; Ferri, S.; Talin, B.; Rosmej, F.; Bureyeva, L.A.; Lisitsa, V.S. Dynamic Stark broadening as the Dicke narrowing effect. Phys. Rev. E 2010, 81, 016406. [CrossRef] [PubMed]

(C) 2017 by the authors. Licensee MDPI, Basel, Switzerland. This article is an open access article distributed under the terms and conditions of the Creative Commons Attribution (CC BY) license (http:/ / creativecommons.org/licenses/by/4.0/). 